\title{
Deferasirox (ExJade): A Fluorescent Pro-Chelator Active Against Antibiotic Resistant Bacteria
}

Adam C. Sedgwick, ${ }^{b, \ddagger}$ Kai-Cheng Yan, ${ }^{a, \ddagger}$ Daniel N. Mangel, ${ }^{b}$ Ying Shang, ${ }^{a}$ Axel Steinbrueck, ${ }^{b}$ HaiHao Han, ${ }^{\text {a }}$ James T. Brewster II, ${ }^{b}$ Xi-Le Hu, ${ }^{a}$ Dylan W. Snelson, ${ }^{b}$ Vincent M. Lynch, ${ }^{b}$ He Tian, ${ }^{a}$ Xiao-Peng $\mathrm{He}^{,{ }^{a},{ }^{*}}$ and Jonathan L. Sessler ${ }^{\mathrm{b},{ }^{*}}$

${ }^{a}$ Key Laboratory for Advanced Materials and Joint International Research Laboratory of Precision Chemistry and Molecular Engineering, Feringa Nobel Prize Scientist Joint Research Center, School of Chemistry and Molecular Engineering, East China University of Science and Technology, 130 Meilong Rd., Shanghai 200237, China;

${ }^{b}$ Department of Chemistry, The University of Texas at Austin, 105 East 24th Street-A5300, Austin, Texas 78712-1224, United States

Abstract: Deferasirox, ExJade, an FDA-approved treatment for iron overload disorders has been shown to inhibit the growth of both gram-positive and -negative bacteria through iron (Fe(III)) chelation. Modification of the ExJade framework led to the identification of a new fluorescent platform ExPh and ExBT. Functionalization of the phenol moieties on ExBT with phosphate units afforded a ratiometric fluorescent pro-chelator (ExPhos), which was effective in the inhibition of two clinically relevant antibiotic-resistant bacteria, (MRSA (ATCC 43300) and VRE (ATCC 51299)), and allowed the fluorescent imaging of MRSA. Remarkably, this pro-chelation strategy proved selective towards bacteria with no cytotoxicity observed for ExPhos treated A549 cells (72 h incubation). This work represents a new pro-chelator antibiotic strategy that can be modified with a chosen reactive chemical trigger to provide a diagnostic signal in conjunction with a therapeutic response with a potential of minimal offtarget toxicities.

\section{Results and Discussion}

Bacterial evolution, coupled with the global misuse of antibiotic treatments, has led to the emergence of antibiotic resistant bacteria (so-called "superbugs") towards which numerous antibiotics are inactive. ${ }^{1-3}$ This is spawning serious public health concerns, including fears of a potential return to the pre-antibiotic era. ${ }^{4}$ Cost effective strategies for overcoming antibiotic resistance and new agents that operate via novel mechanisms of action may help alleviate some of these concerns. Recently, researchers have begun to exploit siderophore-mediated iron uptake pathways using natural or synthetic Fe(III) chelators in an attempt to interfere with bacterial $\mathrm{Fe}$ (III) acquisition and augment host "nutritional immunity" antibacterial mechanisms. ${ }^{5-9}$ Here we report an iron chelation-based approach based on the Deferasirox (ExJade) skeleton that inhibits the growth of both gram-positive and -negative bacteria through Fe(III) chelation. The unique inherent fluorescent nature of the ExJade scaffold also 
allows for the fluorescence-based optical imaging of Methicillin-resistant Staphylococcus aureus MRSA.

Recent efforts with the FDA-approved iron chelator ExJade have demonstrated its ability to act as a chemotherapeutic, insecticide, and antifungal agent; however, minimal efforts apparently have been devoted to exploring its potential as an antimicrobial agent. ${ }^{10-14} \mathrm{We}$ were thus keen to explore whether ExJade and its derivatives might provide a platform for the development of new antimicrobial agents. Specifically, we sought to test whether ExJade alone or after suitable functionalization would display attractive antimicrobial properties. With this goal in mind, ExJade and its derivatives were prepared (Fig. 1; cf. SI for synthesis).

(a)

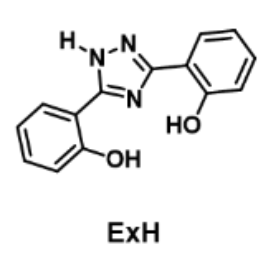

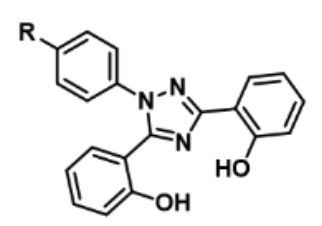

ExJade $(\mathrm{R}=\mathrm{COOH})$ $\operatorname{ExPh}(\mathrm{R}=\mathrm{H})$

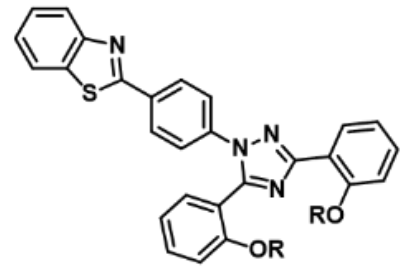

$\operatorname{ExBT}(\mathrm{R}=\mathrm{H})$

$\operatorname{ExBT-OMe}(\mathrm{R}=\mathrm{Me})$

ExPhos $\left(\mathrm{R}=\mathrm{O}=\mathrm{P}(\mathrm{OH})_{2}\right)$

(b)

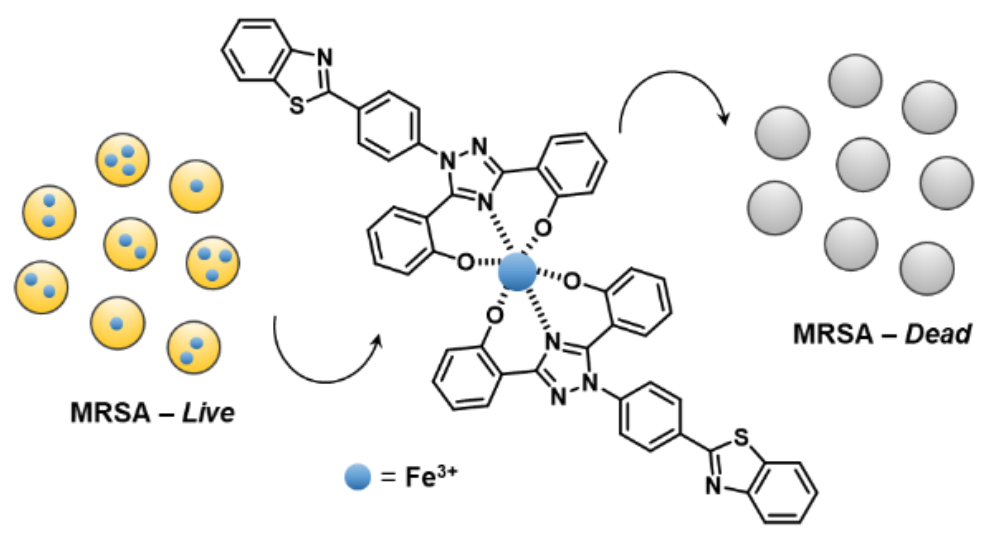

Figure 1 - (a) Structure of the ExJade-based derivatives developed in this study. (b) Schematic illustration of the antimicrobial effect of ExBT for Methicillin-resistant Staphylococcus aureus (MRSA), a clinically prevalent superbug, through the Fe(III)chelating mechanism.

With the above goal in mind, several new ExJade derivatives, designed to control solubility and modulate the intrinsic electronic features, namely ExPh, ExPh-OMe, ExH, ExBT, ExBTOMe and ExPhos (Fig. 1), were prepared (cf. ESI for synthesis). Unexpectedly, two of these derivatives, ExPh and ExBT, were found to display aggregation induced emission (AIE)-like properties in aqueous media (Figure 2). ${ }^{15-16}$ Specifically, insoluble aggregates (as confirmed by DLS; cf. SI - Fig. S1) formed in water, leading to a strong fluorescence emission with a large Stokes shift (> $150 \mathrm{~nm}$ ). Single crystal X-ray diffraction (XRD) analysis for ExPh and ExBT revealed strong intramolecular and intermolecular hydrogen bonding interactions, which is indicative of an excited state proton transfer based system (Figure $2 a$ and $2 b$ ). ${ }^{17,18-25}$ Further analysis of the crystal packing diagram revealed that both ExPh and ExBT exists in a slipstacking orientation, which is known to augment rigidity in AlEgens since it serves to restrict intramolecular rotation (RIR) and enhance the fluorescence-based emission intensity (See ESI 
- Fig. S2-5). ${ }^{26-27}$ In marked contrast to what is seen in aqueous environments, solutions of ExPh and ExBT in most common organic solvents (See SI Fig. S6-9-for solvents tested) display no appreciable emission.
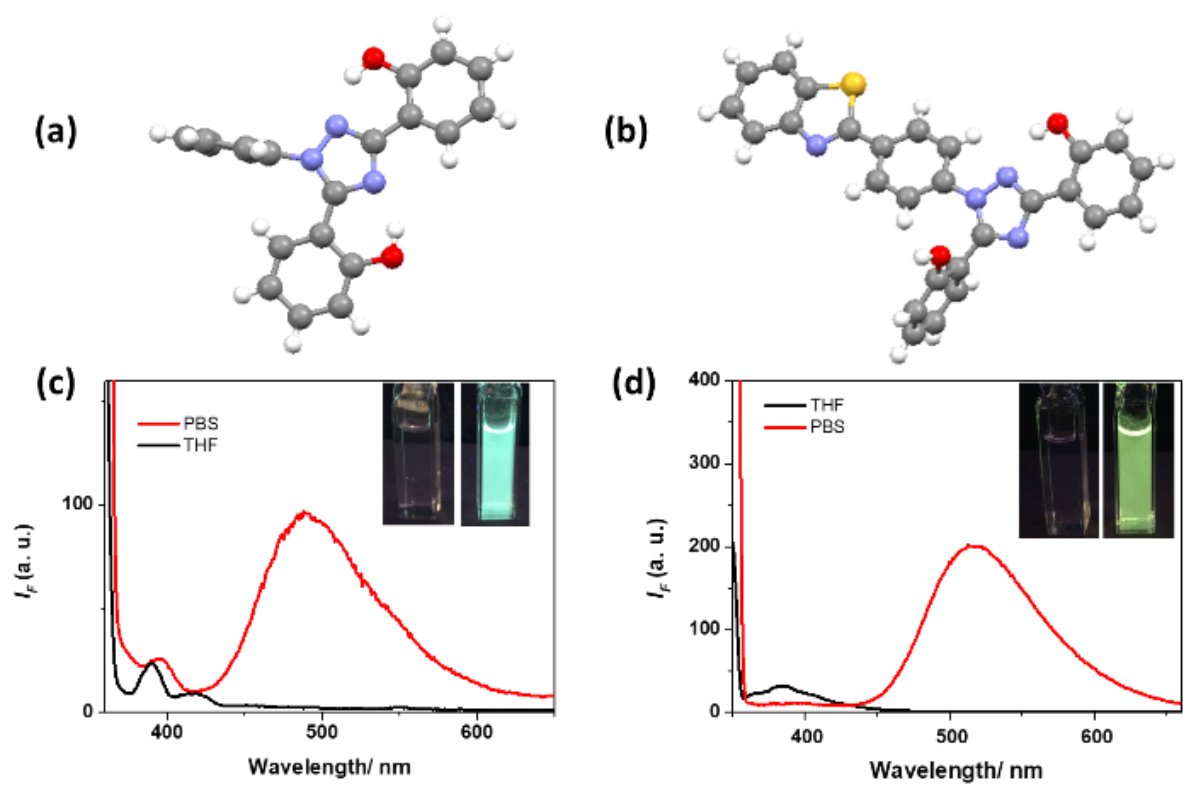

Figure 2 - (a) Ball and stick crystal structure of ExPh. (b) Ball and stick crystal structure of ExBT. (c) Fluorescence spectra of ExPh $(10 \mu \mathrm{M})$ in THF and PBS ( $\mathrm{pH}=7.40) ; \lambda_{\mathrm{ex}}=300 \mathrm{~nm}$. (d) Fluorescence spectra of ExBT $(10 \mu \mathrm{M})$ in THF and PBS $(\mathrm{pH}=7.40)$; $\lambda_{\text {ex }}=350 \mathrm{~nm}$. CCDC 1967984 (ExPh); CCDC 1967981 (ExBT).

In an attempt to elucidate in greater detail the origins of the fluorescence displayed by ExPh and ExBT under conditions favoring aggregation, the derivatives, ExPh-OMe, ExBT-OMe and non- $N$ phenyl functionalized ExH, were studied. ${ }^{22,23}$ In both, aqueous and organic media, ExH was found to display strong fluorescence. This led us to believe the free rotation of the Ph unit effectively quenches the excited state fluorescence of ExPh and ExBT, a phenomenon known as the free rotor effect (see SI - Figs. S10-11). ${ }^{26}$ Upon blocking each phenol unit present on ExPh and ExBT with a methyl group (to give ExPh-OMe and ExBT-OMe), a significant blue-shift in the emission maximum was seen (cf. SI- Fig. S12-15). Such changes in the emission features are consistent with the contention that the free form of the phenol is required for efficient ESIPT. $^{17}$

No significant fluorescence quenching was seen upon changing the solvents in which ExPhOMe and ExBT-OMe were analyzed - DMSO, PhMe, THF, DCM, acetone, MeCN, MeOH and PBS. On this basis we suggest that the ESIPT process is heavily affected by the free rotor effect. ${ }^{28-29}$ In addition, significant solvatochromism was observed for both ExPh-OMe and ExBT-OMe, which leads us to suggest that a type of intramolecular charge transfer (ICT) process $^{30}$ is additionally involved in mediating the fluorescence features of these ExJade derivatives. This putative ICT phenomenon has been observed in structurally similar $N$ phenylpyrrole analogues. ${ }^{31-32}$ Further support for the proposed aqueous induced aggregation mechanism invoked in the case of ExPh and ExBT-OMe came from studies of the parent system, ExJade. In the solid-state ExJade fluoresces; ${ }^{33}$ however, it does not do so in aqueous solution, presumably as the result of its good solubility at neutral pH (See SI - Figure S16-17). 
In the event, ExPh and ExBT proved to be highly emissive in aqueous media, making these species of interest in terms of the potential optical imaging of bacteria and other organisms.

Metal chelators, including ExJade, have begun to raise safety concerns owing to their offtarget metal chelation, which can result in unwanted toxicities. ${ }^{34-35}$ This has led to explorations of protected versions of chelators, often referred to as "pro-chelators". ${ }^{36-40}$ These molecules are designed so in the presence of a disease-related stimulus, the metal binding site will be unmasked and that metal chelation will occur at the desired location. To the extent it is achieved, this high level of therapeutic precision serves to minimalize systemic toxicity. Nearly without exception, metal chelators and pro-chelators that have been studied in the context of biological applications are inherently non-fluorescent. As a result, the addition or conjugation of a fluorophore is necessary to visualize their cellular location. A related approach involves attaching a fluorophore to a pro-chelator that serves to report on the unmasking process.

We thus envisaged the inherent fluorescence of ExJade derivatives provides a unique multifunctional "molecular platform" that can be used to develop a fluorescence responsive pro-chelator active against antibiotic resistant bacteria. This system permits both detection and treatment with little synthetic investment. As a result, this strategy was explored using ExBT, which displayed optical characteristics deemed suitable for fluorescence imaging ( $\lambda_{\text {ex }}>$ $350 \mathrm{~nm}$ ). Phosphate is an essential nutrient for bacterial growth and phosphatases are expressed in a range of bacteria. ${ }^{41-43}$ ExPhos was thus developed to act as a phosphataseresponsive pro-chelator active against antibiotic resistant bacteria with minimal off-target toxicities. The pro-chelator function was not expected to manifest in the case of the control system, ExBT-OMe.

As expected and previously seen for ExJade, ${ }^{44}$ the addition of increasing concentrations of $\mathrm{Fe}(\mathrm{III})$ (as the $\mathrm{FeCl}_{3}$ salt) to both ExBT $(15 \mu \mathrm{M})$ and ExJade $(15 \mu \mathrm{M})$ led to a gradual increase in their respective UV-Vis absorption intensities, along with a color change from clear to purple (Fig. S18) Fluorescence experiments revealed a concentration-dependent quenching of the ExBT fluorescence (Fig. 3a), a common observation for Fe(III) chelation. ${ }^{45}$ No change in the UV-Vis absorption or fluorescence was seen for either ExBT-OMe or ExPhos (Fig. 3b-c) (Fig. S18). This is consistent with the design expectation that a free phenol is need to chelate Fe(III) well. ${ }^{44}$

In the presence of alkaline phosphatase (ALP), a dose dependent change in fluorescence emission was observed ( 0 - $64 \mathrm{U})$, with a final fluorescence emission profile analogous to that of ExBT (Fig. 3d). This ratiometric change in fluorescence emission was ascribed to the gradual and stepwise dephosphorylation of ExPhos, which was confirmed by LC-MS analysis (See ESI - Figs. S19-S29). ExPhos solutions exposed to ALP (64 U ALP) were subsequently treated with increasing concentrations of $\mathrm{Fe}$ (III) (as the $\mathrm{FeCl}_{3}$ salt). As expected for a system capable of chelating Fe(III), a quenching of the fluorescence intensity was observed (Fig. 3f). Based on these findings, we considered it likely that ExPhos could serve as an ALP-responsive Fe(III) prochelator. 

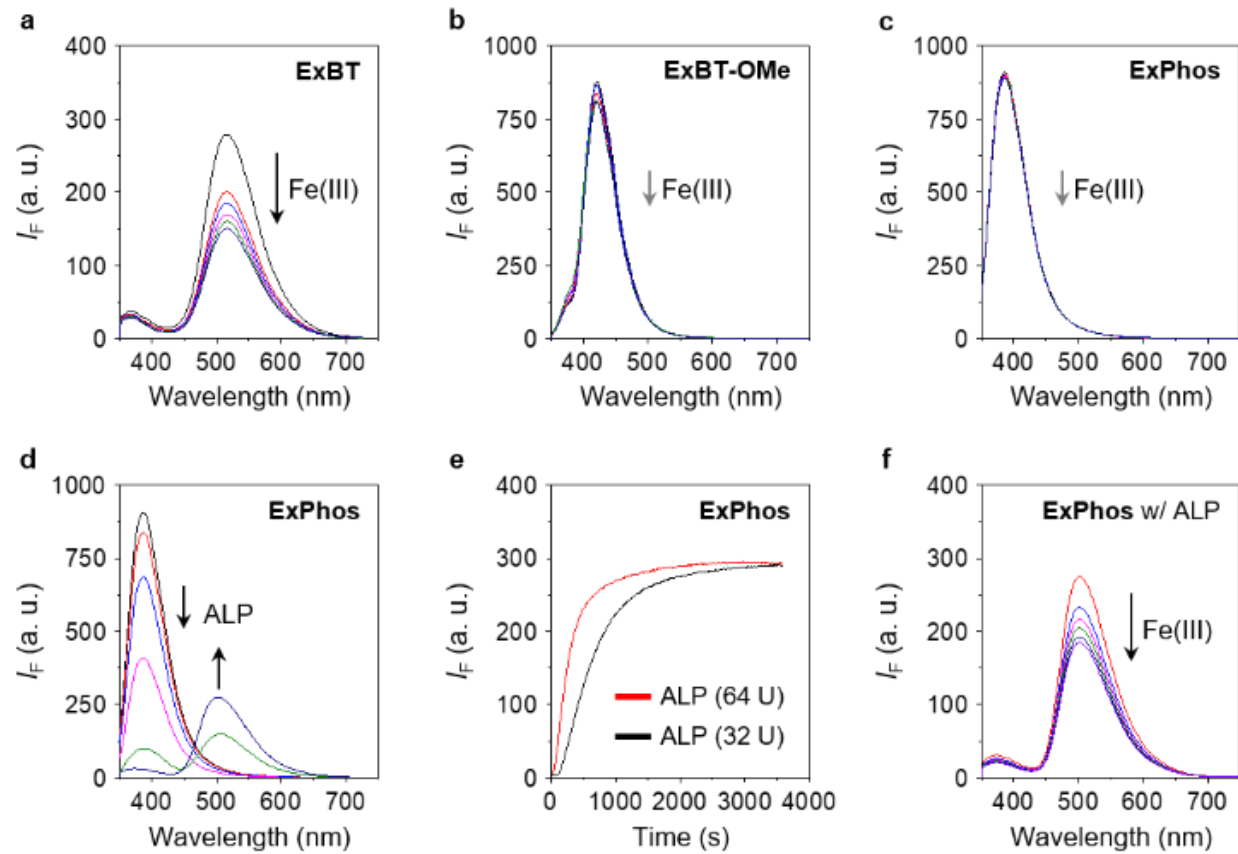

Figure 3 - Fluorescence spectra of (a) ExBT $(15 \mu \mathrm{M})$ ), (b) ExBT-OMe, and (c) ExPhos (15 $\mu \mathrm{M})$ recorded upon exposure to increasing $\mathrm{Fe}(\mathrm{III})$ concentrations $\left(0-15 \mu \mathrm{M}\right.$ as the $\mathrm{FeCl}_{3}$ salt). (d) Fluorescence changes of ExPhos (15 $\left.\mu \mathrm{M}\right)$ with increasing alkaline phosphatase (ALP, 0-64 U). (e) Time-dependent change in the fluorescence-emission intensity at $505 \mathrm{~nm}$ observed when solutions containing ExPhos (15 $\mu \mathrm{M}$ ) and ALP (two concentrations) were excited at $320 \mathrm{~nm}$. (f) Fluorescence spectra of ExPhos $(15 \mu \mathrm{M})$ preincubated with $64 \mathrm{U}$ of ALP recorded as a function of increasing Fe(III) $(0-15 \mu \mathrm{M})$. All measurements were carried out in deionized water.

With the photophysical properties identified, the bacterial inhibitory effect of the present ExJade derivatives were then evaluated. Initial tests were focused on two Gram-negative, ESKAPE bacteria, ${ }^{46}$ Pseudomonas aeruginosa (ATCC 27853) and Klebsiella pneumoniae (ATCC 13883). Arbekacin, an FDA-approved aminoglycoside-based antibiotic, was used as positive control. ExJade, ExPh, ExBT, and ExPhos were found capable of inhibiting the growth of these two bacterial strains in a statistically significant manner (See ESI - Figs. S30-31).

More importantly, two clinically relevant gram-positive, drug-resistant bacteria, methicillinresistant Staphylococcus aureus (MRSA, ATCC 43300) and vancomycin-resistant Enterococcus faecalis (VRE, ATCC 51299) with high ALP activity (Fig. S32) were then used to evaluate further the therapeutic potential of these ExJade derivatives. Again, arbekacin was used as a positive control. To our delight, strong inhibitory activities were observed for the suppression of these "superbugs" with a potency comparable to that of arbekacin (Fig. 4). The observed inhibition of VRE is of particular significance since there are few clinically available treatments for this multidrug-resistant bacterium.

The pre-incubation with increasing concentrations of Fe(III) led to a corresponding decrease in the inhibitory activity (Figs. S33, S34) including no appreciable inhibitory activity seen for either MRSA or Pseudomonas aeruginosa in the case of the control system ExBT-OMe. This observation confirms the Fe(III) chelation dependent inhibitory activity. Due to the Fe(III) 
chelation dependent nature of the ExJade derivatives and to illustrate their potential "real life" utility, the inhibitory activity of ExBT and ExPhos against MRSA were evaluated in a blood containing medium consisting of agar and defibrillated amniotic blood. ${ }^{47}$ Remarkably, no adverse effect was seen, with the inhibitory activity of ExBT and ExPhos remaining strong (Fig. S35). In addition, no significant cytotoxicity was observed for ExPhos towards A549 cells, providing an initial indication that the ExJade-based pro-chelation approach reported here may prove selective towards bacteria (See ESI - Fig. S36).

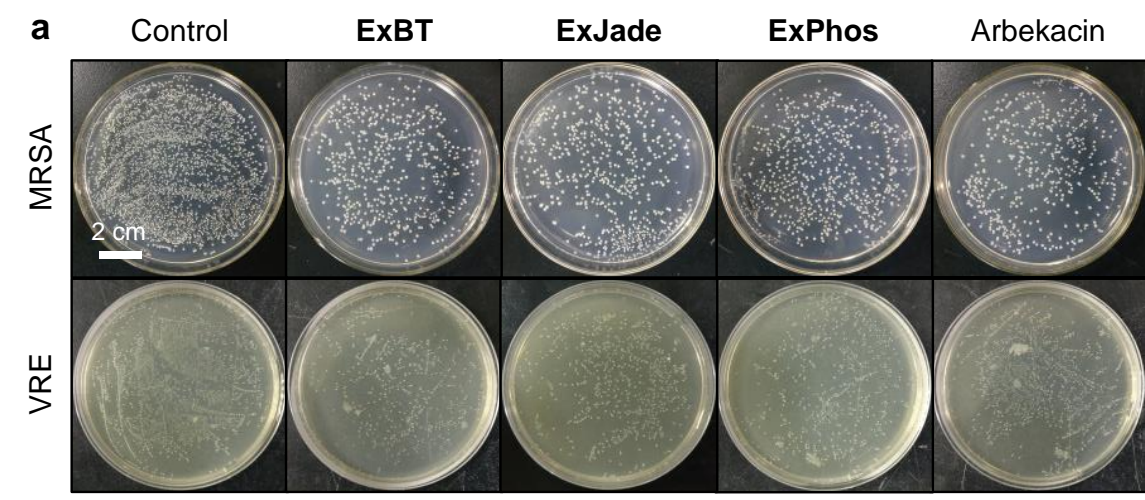

b

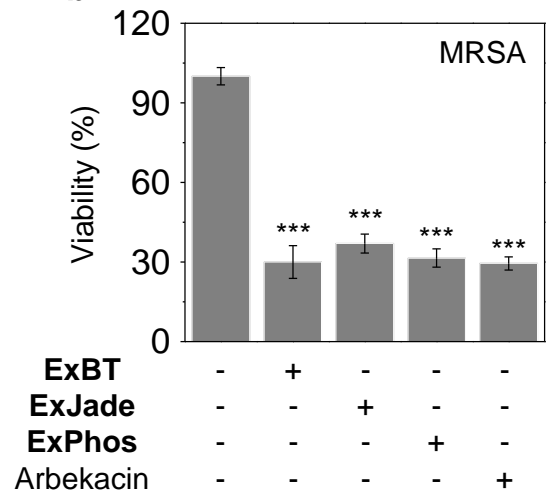

c

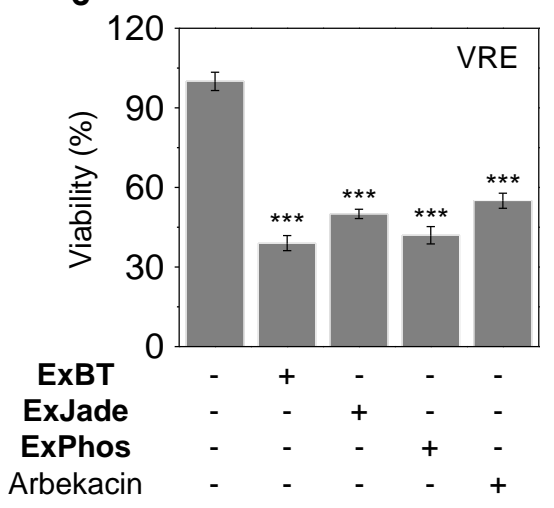

Figure 4 - (a) MRSA (ATCC 43300) colonies on Luria-Bertani (LB) tryptone agar plates and VRE (ATCC 51299) colonies on BrainHeart Infusion (BHI) tryptone agar plates before and after treatment with ExBT, ExJade, ExPhos, and arbekacin (15 $\mu \mathrm{M})$. Cell viability of (b) MRSA (ATCC 43300) and (c) VRE (ATCC 51299) before and after treatment with ExBT, ExJade, ExPhos, and arbekacin $(15 \mu \mathrm{M})$.

Lastly, to determine whether the present ExJade derivatives might have a role to play as fluorescence imaging agents and theranostic agents, MRSA was incubated separately with ExBT and ExPhos; both compounds produced bright fluorescence in bacteria (Fig. 5a) Interestingly, the pre-treatment of MRSA with Phosphatase Inhibitor cocktail A (sodium fluoride, sodium pyrophosphate, $\beta$-glycerophosphate and sodium orthovanadate) led to an inhibitor concentration dependent decrease in the fluorescence emission intensity (Fig. $5 \mathrm{a}$ and Fig. 5b). This finding further confirms our contention that ExPhos is a pro-chelator that is activated under conditions of use via a phosphatase-mediated pathway. 

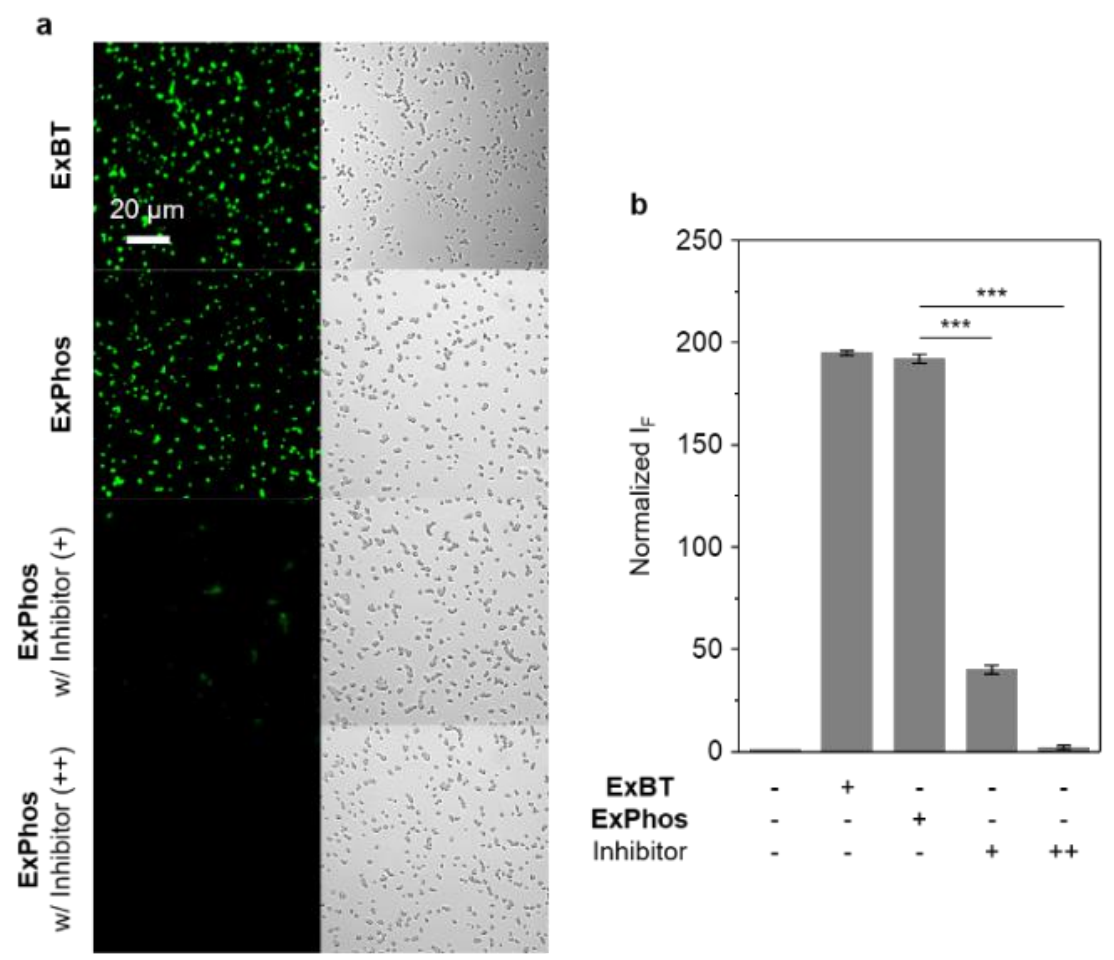

Figure 5 - (a) Confocal Laser-Scanning Microscope (CLSM) images (left: fluorescence images; right: bright-field images) of MRSA (ATCC 43300) treated with ExBT $(40 \mu \mathrm{M})$, ExPhos $(40 \mu \mathrm{M})$, or ExPhos pretreated with different concentrations of a phosphatase inhibitor cocktail A (+ $50 \mu \mathrm{M}$ sodium fluoride, $10 \mu \mathrm{M}$ sodium pyrophosphate, $10 \mu \mathrm{M}$-glycerophosphate, and $10 \mu \mathrm{M}$ sodium orthovanadate; ++: $100 \mu \mathrm{M}$ sodium fluoride, $20 \mu \mathrm{M}$ sodium pyrophosphate, $20 \mu \mathrm{M} \beta$-glycerophosphate and $20 \mu \mathrm{M}$ sodium orthovanadate). (b) Normalized fluorescence intensities of the fluorescent imaged systems. ${ }^{* * *} \mathrm{P}<0.001$.

In summary, ExJade, an FDA-approved treatment for iron overload disorders has been identified to inhibit the growth of both gram-positive and -negative bacteria through iron (Fe(III)) chelation. The modification of ExJade revealed a new fluorescent framework that was shown effective in the inhibition of two clinically relevant antibiotic-resistant bacteria (MRSA (ATCC 43300) and VRE (ATCC 51299)), while also displaying an ability to image MRSA. This work highlights a pro-chelator strategy that exploits the unique inherent fluorescence nature of the ExJade scaffold while showing how this core can be modified with a chosen reactive chemical trigger to provide a diagnostic signal in conjunction with a therapeutic response.

\section{Acknowledgements}

The authors thank the National Natural Science Foundation of China (Nos. 21788102, 91853201, 21722801, 81673489 and 31871414), the Shanghai Municipal Science and Technology Major Project (No. 2018SHZDZX03), the International Cooperation Program of Shanghai Science and Technology Committee (No. 17520750100) and the Fundamental Research Funds for the Central Universities (222201717003) for financial support. The work in Austin was supported by the National Institutes of Health (R01 GM103790 to J.L.S.) and the Robert A. Welch Foundation (F-0018). 


\section{References}

(1) Wright, P. M.; Seiple, I. B.; Myers, A. G., The Evolving Role of Chemical Synthesis in Antibacterial Drug Discovery. Angew. Chem. Int. Ed. 2014, 53 (34), 8840-8869.

(2) von Bubnoff, A., Seeking new antibiotics in nature's backyard. Cell. 2006, 127 (5), 867-869.

(3) Peeters, P.; Ryan, K.; Karve, S.; Potter, D.; Baelen, E.; Rojas-Farreras, S.; Rodriguez-Bano, J., The impact of initial antibiotic treatment failure: real-world insights in patients with complicated, health care-associated intra-abdominal infection. Infect. Drug Resist. 2019, 12, 329-343.

(4) Rugina, S., Resistance to Antimicrobians - A Global Problem with Sectoral Resolution. J. Crit. Care Med. 2018, 4 (2), 47-49.

(5) Negash, K. H.; Norris, J. K. S.; Hodgkinson, J. T., Siderophore-Antibiotic Conjugate Design: New Drugs for Bad Bugs? Molecules. 2019, 24 (18). DOI: 10.3390/molecules24183314

(6) Thompson, M. G.; Corey, B. W.; Si, Y. Z.; Craft, D. W.; Zurawski, D. V., Antibacterial Activities of Iron Chelators against Common Nosocomial Pathogens. Antimicrob. Agents Chemother. 2012, 56 (10), 5419-5421.

(7) Miller, M. J.; Malouin, F., Microbial Iron Chelators as Drug Delivery Agents - The Rational Design and Synthesis of Siderophore-Drug Conjugates. Acc. Chem. Res. 1993, 26 (5), 241-249.

(8) Mislin, G. L. A.; Schalk, I. J., Siderophore-dependent iron uptake systems as gates for antibiotic Trojan horse strategies against Pseudomonas aeruginosa. Metallomics 2014, 6 (3), 408-420.

(9) Hennigar, S. R.; McClung, J. P., Nutritional Immunity: Starving Pathogens of Trace Minerals. Am. J. Lifestyle Med. 2016, 10 (3), 170-173.

(10) Kielar, F.; Wang, Q.; Boyle, P. D.; Franz, K. J., A boronate prochelator built on a triazole framework for peroxide-triggered tridentate metal binding. Inorg. Chim. Acta 2012, 393, 294303.

(11) Loza-Rosas, S. A.; Vazquez-Salgado, A. M.; Rivero, K. I.; Negron, L. J.; Delgado, Y.; Benjamin-Rivera, J. A.; Vazquez-Maldonado, A. L.; Parks, T. B.; Munet-Colon, C.; Tinoco, A. D., Expanding the Therapeutic Potential of the Iron Chelator Deferasirox in the Development of Aqueous Stable Ti(IV) Anticancer Complexes. Inorg. Chem. 2017, 56 (14), 7788-7802.

(12) Puri, S.; Kumar, R.; Rojas, I. G.; Salvatori, O.; Edgerton, M., Iron Chelator Deferasirox Reduces Candida albicans Invasion of Oral Epithelial Cells and Infection Levels in Murine Oropharyngeal Candidiasis. Antimicrob. Agents Chemother. 2019, 63 (4), DOI: 10.1128/AAC.02152-18

(13) Lui, G. Y. L.; Obeidy, P.; Ford, S. J.; Tselepis, C.; Sharp, D. M.; Jansson, P. J.; Kalinowski, D. S.; Kovacevic, Z.; Lovejoy, D. B.; Richardson, D. R., The Iron Chelator, Deferasirox, as a Novel Strategy for Cancer Treatment: Oral Activity Against Human Lung Tumor Xenografts and Molecular Mechanism of Action. Mol. Pharmacol. 2013, 83 (1), 179-190. 
(14) Gupta, P.; Jat, K.; Solanki, V. S.; Shrivastava, R., Synthesis and Antimicrobial Activity of some New N'-Arylidene-4-(3,5-Bis(2-Hydroxyphenyl)-1H-1,2,4-Triazole-1-yl) Benzohydrazides. Indian J. Heterocycl. Chem. 2017, 27 (2), 151-156.

(15) Hong, Y. N.; Lam, J. W. Y.; Tang, B. Z., Aggregation-induced emission: phenomenon, mechanism and applications. Chem. Commun. 2009, (29), 4332-4353.

(16) Qian, J.; Tang, B. Z., AIE Luminogens for Bioimaging and Theranostics: from Organelles to Animals. Chem. 2017, 3 (1), 56-91.

(17) Sedgwick, A. C.; Wu, L. L.; Han, H. H.; Bull, S. D.; He, X. P.; James, T. D.; Sessler, J. L.; Tang, B. Z.; Tian, H.; Yoon, J., Excited-state intramolecular proton-transfer (ESIPT) based fluorescence sensors and imaging agents. Chem. Soc. Rev. 2018, 47 (23), 8842-8880.

(18) Zhao, J. F.; Chen, J. S.; Liu, J. Y.; Hoffmann, M. R., Competitive excited-state single or double proton transfer mechanisms for bis-2,5-(2-benzoxazolyl)-hydroquinone and its derivatives. Phys. Chem. Chem. Phys. 2015, 17 (18), 11990-11999.

(19) Hao, Y. C.; Chen, Y., Excited-state intramolecular single and double proton transfer emission of 2,5-bis(benzoxazol-2-yl)thiophene-3,4-diol. Dyes Pigm. 2016, 129, 186-190.

(20) Tang, K. C.; Chen, C. L.; Chuang, H. H.; Chen, J. L.; Chen, Y. J.; Lin, Y. C.; Shen, J. Y.; Hu, W. P.; Chou, P. T., A Genuine Intramolecular Proton Relay System Undergoing Excited-State Double Proton Transfer Reaction. J. Phys. Chem. Lett. 2011, 2 (24), 3063-3068.

(21) Peng, C. Y.; Shen, J. Y.; Chen, Y. T.; Wu, P. J.; Hung, W. Y.; Hu, W. P.; Chou, P. T., Optically Triggered Stepwise Double-Proton Transfer in an Intramolecular Proton Relay: A Case Study of 1,8-Dihydroxy-2-naphthaldehyde. J. Am. Chem. Soc. 2015, 137 (45), 14349-14357.

(22) Sahu, S.; Das, M.; Bharti, A. K.; Krishnamoorthy, G., Proton transfer triggered proton transfer: a self-assisted twin excited state intramolecular proton transfer. Phys. Chem. Chem. Phys. 2018, 20 (42), 27131-27139.

(23) Das, M.; Sahu, S.; Krishnamoorthy, G., Tweaking the proton transfer triggered proton transfer of 3,5-bis(2-hydroxyphenyl)-1H-1,2,4-triazole. Phys. Chem. Chem. Phys. 2019, 21 (28), 15669-15677.

(24) Sedgwick, A. C.; Dou, W. T.; Jiao, J. B.; Wu, L. L.; Williams, G. T.; Jenkins, A. T. A.; Bull, S. D.; Sessler, J. L.; He, X. P.; James, T. D., An ESIPT Probe for the Ratiometric Imaging of Peroxynitrite Facilitated by Binding to A beta-Aggregates. J. Am. Chem. Soc. 2018, 140 (43), 14267-14271.

(25) Wu, L. L.; Han, H. H.; Liu, L. Y.; Gardiner, J. E.; Sedgwick, A. C.; Huang, C. S.; Bull, S. D.; He, X. P.; James, T. D., ESIPT-based fluorescence probe for the rapid detection of peroxynitrite 'AND' biological thiols. Chem. Commun. 2018, 54 (80), 11336-11339.

(26) Lou, Z. R.; Hou, Y. Q.; Chen, K. P.; Zhao, J. Z.; Ji, S. M.; Zhong, F. F.; Dede, Y.; Dick, B., Different Quenching Effect of Intramolecular Rotation on the Singlet and Triplet Excited States of Bodipy. J. Phys. Chem. C 2018, 122 (1), 185-193. 
(27) Sivalingam, S.; Debsharma, K.; Dasgupta, A.; Sankararaman, S.; Prasad, E., Effect of SlipStack Self-Assembly on Aggregation-Induced Emission and Solid-State Luminescence in 1,3Diarylpropynones. Chempluschem 2019, 84 (4), 392-402.

(28) Vollmer, F.; Rettig, W., Fluorescence loss mechanism due to large-amplitude motions in derivatives of 2,2'-bipyridyl exhibiting excited-state intramolecular proton transfer and perspectives of luminescence solar concentrators. Journal of Photochemistry and Photobiology a-Chemistry 1996, 95 (2), 143-155.

(29) Kim, S.; Seo, J.; Park, S. Y., Torsion-induced fluorescence quenching in excited-state intramolecular proton transfer (ESIPT) dyes. J. Photochem. Photobio. A Chem. 2007, 191 (1), 19-24.

(30) Pannipara, M.; Asiri, A. M.; Alamry, K. A.; Arshad, M. N.; El-Daly, S. A., Synthesis, spectral behaviour and photophysics of donor-acceptor kind of chalcones: Excited state intramolecular charge transfer and fluorescence quenching studies. Spectrochim. Acta A. 2015, 136, 1893-1902.

(31) Yoshihara, T.; Druzhinin, S. I.; Demeter, A.; Kocher, N.; Stalke, D.; Zachariasse, K. A., Kinetics of intramolecular charge transfer with $\mathrm{N}$-phenylpyrrole in alkyl cyanides. J. Phys. Chem. A 2005, 109 (8), 1497-1509.

(32) Yoshihara, T.; Galievsky, V. A.; Druzhinin, S. I.; Saha, S.; Zachariasse, K. A., Singlet excited state dipole moments of dual fluorescent $\mathrm{N}$-phenylpyrroles and 4(dimethylamino)benzonitrile from solvatochromic and thermochromic spectral shifts. Photochem. Photobiol. Sci. 2003, 2 (3), 342-353.

(33) Zhang, Y. J.; Yang, H. Y.; Ma, H. L.; Bian, G. F.; Zang, Q. G.; Sun, J. W.; Zhang, C.; An, Z. F.; Wong, W. Y., Excitation Wavelength Dependent Fluorescence of an ESIPT Triazole Derivative for Amine Sensing and Anti-Counterfeiting Applications. Angew. Chem. Int. Ed. 2019, 58 (26), 8773-8778.

(34) Diaz-Garcia, J. D.; Gallegos-Villalobos, A.; Gonzalez-Espinoza, L.; Sanchez-Nino, M. D.; Villarrubia, J.; Ortiz, A., Deferasirox nephrotoxicity-the knowns and unknowns. Nat. Rev. Nephrol. 2014, 10 (10), 574-586.

(35) Kontoghiorghes, G. J., Turning a blind eye to deferasirox's toxicity? Lancet 2013, 381 (9873), 1183-1184.

(36) Hyman, L. M.; Franz, K. J., A cell-permeable fluorescent prochelator responds to hydrogen peroxide and metal ions by decreasing fluorescence. Inorg. Chim. Acta 2012, 380, 125-134.

(37) Zaengle-Barone, J. M.; Jackson, A. C.; Besse, D. M.; Becken, B.; Arshad, M.; Seed, P. C.; Franz, K. J., Copper Influences the Antibacterial Outcomes of a beta-Lactamase-Activated Prochelator against Drug-Resistant Bacteria. ACS Infect. Dis. 2018, 4 (6), 1019-1029.

(38) Bakthavatsalam, S.; Sleeper, M. L.; Dharani, A.; George, D. J.; Zhang, T.; Franz, K. J., Leveraging -Glutamyl Transferase To Direct Cytotoxicity of Copper Dithiocarbamates against Prostate Cancer Cells. Angew. Chem. Int. Ed. 2018, 57 (39), 12780-12784. 
(39) Franks, A. T.; Franz, K. J., A prochelator with a modular masking group featuring hydrogen peroxide activation with concurrent fluorescent reporting. Chem. Commun. 2014, 50 (77), 11317-11320.

(40) Wang, Q.; Franz, K. J., Stimulus-Responsive Prochelators for Manipulating Cellular Metals. Acc. Chem. Res. 2016, 49 (11), 2468-2477.

(41) Sajid, A.; Arora, G.; Singhal, A.; Kalia, V. C.; Singh, Y., Protein Phosphates of Pahtogenic Bacteria: Role in Physiology and Virulence. Annu. Rev. Microbiology, 2015, 69, 527-547.

(42) Braibant, M.; Content, J., The cell surface associated phosphatase activity of Mycobacterium bovis BCG is not regulated by environmental inorganic phosphate. FEMS Microbiol. Lett. 2001, 195 (2), 121-126.

(43) Kriakov, J.; Lee, S. H.; Jacobs, W. R., Identification of a regulated alkaline phosphatase, a cell surface-associated lipoprotein, in Mycobacterium smegmatis. J. Bacteriol. 2003, 185 (16), 4983-4991.

(44) Steinhauser, S.; Heinz, U.; Bartholoma, M.; Weyhermuller, T.; Nick, H.; Hegetschweiler, K., Complex formation of ICL670 and related ligands with Fe-III and Fe-II. Eur. J. Inorg. Chem. 2004, (21), 4177-4192.

(45) Qu, X. Y.; Liu, Q.; Ji, X. N.; Chen, H. C.; Zhou, Z. K.; Shen, Z., Enhancing the Stokes' shift of BODIPY dyes via through-bond energy transfer and its application for Fe3+-detection in live cell imaging. Chemical Communications 2012, 48 (38), 4600-4602.

(46) Mulani, M. S.; Kamble, E. E.; Kumkar, S. N.; Tawre, M. S.; Pardesi, K. R., Emerging Strategies to Combat ESKAPE Pathogens in the Era of Antimicrobial Resistance: A Review. Front. Microbiol. 2019, 10. DOI: 10.3389/fmicb.2019.00539

(47) Filipowicz, N.; Kaminski, M.; Kurlenda, J.; Asztemborska, M.; Ochocka, J. R., Antibacterial and antifungal activity of juniper berry oil and its selected components. Phytother. Res. 2003, $17(3), 227-231$. 